\title{
HÁ INFLUÊNCIA DE CARACTERÍSTICAS DO TOP MANAGEMENT TEAM NA ESTRUTURA DE CAPITAL DAS EMPRESAS FINANCEIRAS?
}

\author{
(D) Paula de Souza Michelon ${ }^{1}$ (D) ${\text { Rogério João } \text { Lunkes }^{2} \text { iD }}^{\text {Antonio Cezar Bornia }}{ }^{3}$ \\ ${ }^{1}$ Doutora em Engenharia de Produção, Universidade Federal de Santa Catarina - UFSC. Florianópolis - SC, Brasil.pauladesouza1604@gmail.com \\ ${ }^{2}$ Doutor em Engenharia de Produção, Universidade Federal de Santa Catarina - UFSC. Florianópolis - SC, Brasil.rogeriolunkes@hotmail.com \\ ${ }^{3}$ Doutor em Engenharia de Produção, Universidade Federal de Santa Catarina - UFSC. Florianópolis - SC, Brasil. cezar.bornia@gmail.com
}

\section{Resumo}

Objetivo: analisar a relação entre as características do Top Management Team e a estrutura de capital das empresas financeiras.

Originalidade: a Teoria dos Escalões Superiores (TES) preconiza que os valores, as experiências e as personalidades do Top Management Team influenciam nas escolhas estratégicas das empresas. Deste modo, espera-se que diferentes equipes de gestores escolham estruturas de capital que melhor atendam às necessidades de diferentes estratégias. Método: Foram testadas hipóteses de modelos teóricos por meio de regressão linear e linear múltipla. A amostra foi composta por 95 companhias financeiras listadas na Brasil, Bolsa, Balcão em julho de 2019. Os fatores analisados foram: Idade média do TMT, Nível educacional médio do TMT, Tempo médio no cargo do TMT e Nível de capital de terceiros da empresa.

Resultados: A idade média do TMT foi aquela que apresentou relação significativa positiva com o nível de capital de terceiros da empresa. As variáveis tempo médio no cargo e nível médio educacional do TMT apresentaram relação negativa com o endividamento das empresas, mas sem forte significância estatística.

Contribuições: Esta pesquisa contribui para a estratégia das empresas na composição de suas equipes de gestão, uma vez que estão relacionadas com o desempenho das organizações. Do ponto de vista teórico, é capaz de ratificar os pressupostos da Teoria dos Escalões Superiores.

Palavras-chave: Equipe de gestão de topo. Estrutura de capital. Custo do capital. Capital de terceiros.

\section{IS THERE INFLUENCE OF TOP MANAGEMENT TEAM CHARACTERISTICS IN THE CAPITAL STRUCTURE OF FINANCIAL COMPANIES?}

\begin{abstract}
Objective: to analyze the relationship between the characteristics of Top Management Team and the capital structure of financial companies. Originality: The Upper Echelons Theory (UET) advocates that the values, experiences and personalities of Top Management Team influence the strategic choices of companies. In this way, different teams of managers are expected to choose capital structures that best meet the needs of different strategies.

Method: Hhypotheses of theoretical models were tested through linear and multiple linear regression. The sample consisted of 95 financial companies listed in Brazil, Bolsa, Balcão in July 2019. The factors analyzed were: TMT's average age, TMT's average educational level, TMT's average time in the company and the company's third-party capital level.
\end{abstract}

Results: TMT's average age was that which had a significant positive relationship with the company's third party capital level. The variables mean time in office and average educational level of TMT were negatively related to corporate indebtedness, but without strong statistical significance.

Contributions: This research contributes to the strategy of companies in the composition of their management teams, since they are related to the performance of organizations. From the theoretical point of view, it is able to ratify the assumptions of the Upper Echelons Theory.

Keywords: Top management team. Capital structure. Cost of capital. Third-party capital.

\section{¿HAY INFLUENCIA DE LAS CARACTERÍSTICAS DEL EQUIPO DE GESTIÓN EN LA ESTRUCTURA DE CAPITAL DE LAS EMPRESAS FINANCIERAS?}

\section{Resumen}

Objetivo: analizar la relación entre las características del equipo directivo superior y la estructura de capital de las empresas financieras. Originalidad: la Teoría del Nivel Superior (TNS) establece que los valores, las experiencias y las personalidades del equipo de alta dirección influyen en las elecciones estratégicas de las empresas. Por lo tanto, se espera que diferentes equipos de gerentes elijan las estructuras de capital que mejor satisfagan las necesidades de las diferentes estrategias.

Método: Se probaron hipótesis de modelos teóricos mediante regresión lineal y lineal múltiple. La muestra consistió en 95 compañías financieras listadas en Brasil, Bolsa, Balcão en julio de 2019. Los factores analizados fueron: edad promedio de TMT, nivel educativo promedio de TMT, tiempo promedio de TMT en el cargo y nivel de capital de terceros de la compañía.

Resultados: La edad promedio de TMT fue la que tuvo una relación positiva significativa con el nivel de capital de terceros de la compañía. Las variables tiempo medio en el cargo y el nivel educativo promedio de TMT se relacionaron negativamente con el endeudamiento corporativo, pero sin una fuerte significación estadística.

Contribuciones: Esta investigación contribuye a la estrategia de las empresas en la composición de sus equipos de gestión, ya que están relacionadas con el desempeño de las organizaciones. Desde el punto de vista teórico, es capaz de ratificar los supuestos de la Teoría del Nivel Superior.

Palabras clave: Equipo directivo superior. Estructura de capital. Costo de capital. Capital de terceros.

\section{Cite as / Como citar}

American Psychological Association (APA)

Michelon, P. S., Lunkes, R. J., \& Bornia, A. C. (2020). Há influência de características do Top Management Team na estrutura de capital das empresas financeiras? Iberoamerican Journal of Strategic Management (IJSM), 19(2), 87-104. https://doi.org/10.5585/riae.v19i2.16132. 


\section{Introdução}

Hambrick e Mason (1984) postularam que as características do Top Management Team (TMT) e as suas escolhas estratégicas ajudam a explicar o desempenho de uma organização. A premissa da Teoria é que o foco sobre as características da equipe irá produzir explicações mais fortes de resultados organizacionais do que o foco habitual no alto executivo individual (por exemplo, $C E O$ ). Isso porque, liderança de uma organização complexa é uma atividade compartilhada e as cognições, capacidades e interações de toda a equipe afetam o comportamento estratégico (Hambrick, 2007).

A definição de TMT baseia-se no trabalho original de Cyert e March (1963), que se referiram a ela ao tratar da coalizão dominante (Carpenter, Geletkanycz, \& Sanders, 2004). Em nível prático, o estudo de toda uma equipe aumenta a força potencial da teoria para prever, porque o chefe executivo compartilha tarefas e, em certa medida, o poder com outros membros da equipe (Hambrick \& Mason, 1984). Gestores desempenham um papel crítico nas políticas de investimento, políticas financeiras, estratégias organizacionais e desempenho operacional das empresas (Bertrand, \& Schoar, 2003). Nesse sentido, as ferramentas contábeis e de gestão podem ser vistas como um aspecto da estrutura organizacional (Chenhall, 2003; Strauß \& Zecher, 2013) e, em consonância com a Teoria dos Escalões Superiores, sua escolha pode ser influenciada pelas características do TMT.

Os gestores de altos escalões impactam importantes decisões estratégicas relacionadas a atividades como investimentos, formação de alianças estratégicas e internacionalização, sendo que cada uma delas provocará impactos no desempenho (Cabrera-Suárez \& Martín-Santana, 2013, Wang, 2015; Xie, Wang, \& Qi, 2015). As habilidades dos gerentes de alto nível são um fator inobservável que tem sido amplamente negligenciado na pesquisa de estrutura de capital, mas pode ter uma forte influência nas decisões de estrutura de capital das empresas (Matemilola, Bany-Ariffin, Azman-Saini, \& Nassir, 2018). As decisões de financiamento resultam em uma dada estrutura de capital e decisões de financiamento abaixo do ideal podem levar ao fracasso corporativo. Por isso, um dos grandes dilemas para a administração e para os investidores é se existe uma estrutura de capital ideal (Mwangi, Makau, \& Kosimbei, 2014).

Embora pesquisas demonstrem, por exemplo, que diretores financeiros mais novos e gestores de topo com experiências na área de negócios estão associados a sistemas de contabilidade e controle mais inovadores e/ou sofisticados, uma gama de oportunidades promissoras para futuros pesquisas continua aberta (Hiebl, 2014). Poderão ser realizadas contribuições valiosas, abordando o efeito de sistemas adicionais de contabilidade e controle gerencial, além de características de escalões superiores e investigação de variáveis moderadoras (Hiebl, 2014). Nesse contexto, a presente pesquisa objetiva analisar a relação entre as características do Top Management Team e a estrutura de capital das empresas financeiras. Pretende-se explorar o efeito das características dos gestores no nível de endividamento da empresa, ou seja, capital de terceiros. 
Empresas financeiras operam alavancadas, isto é, a participação de capital próprio costuma ser significativamente menor que a participação de capital de terceiros no financiamento de suas operações. Algumas pesquisas procuraram definir alguns determinantes da estrutura de capital bancária (Ahmad, Ariff, \& Skully, 2009; Gropp \& Heider, 2010; Octavia \& Brown, 2010). Chevalier e Scharfstein (1995) e Maksimovic (1998) buscaram explicar a relação entre estratégia e estrutura de capital, mas sem observar a influência das características dos gestores.

Barker e Mueller (2002), Kor (2006) e Chen, Hsu e Huang (2010) avaliaram a associação entre as características dos executivos e a estrutura de capital das empresas, mas com foco no financiamento com pesquisa e desenvolvimento. Hanousek e Shamshur (2011) e Lemmon et al. (2008), entre outros, mostraram empiricamente que fatores específicos da empresa não observados explicam a maior parte da variação transversal na estrutura de capital da empresa. Assim, ratifica-se a importância de se verificar a influência das características do TMT na estrutura de capital das empresas financeiras.

\section{Fundamento teórico}

\subsection{Teoria dos escalões superiores - TES}

A Teoria dos Escalões Superiores (TES) indica que os resultados e processos estratégicos das empresas estão em função das características dos gestores superiores. A principal consideração da Teoria é que as escolhas estratégicas, ao contrário das decisões operacionais, são mais provenientes de fatores comportamentais do que de cálculo mecânico de uma otimização econômica. Como consequência, as escolhas estratégicas comumente possuem uma grande quantidade de componentes comportamentais e, de alguma maneira, refletem as idiossincrasias dos tomadores de decisão. As idiossincrasias incluem sua base cognitiva (conhecimento/suposição sobre eventos futuros, conhecimento de alternativas e conhecimento de consequências de alternativas) e valores (princípios para ordenar consequências ou alternativas) que filtram e enquadram a situação de decisão que os executivos enfrentam e, eventualmente, criam suas percepções (Chuang, Nakatani, \& Zhou, 2007; Chuang, Nakatani, \& Zhou, 2009).

Hambrick e Mason (1984) discutiram que poucas das grandes empresas são administradas por uma única pessoa do alto escalão e, então, uma atenção deve ser dada ao gestor que opera no cume decisório. Mas, a Teoria orienta para a liderança estratégica, considerando que a gestão de uma empresa é tipicamente uma atividade compartilhada e fornece um modelo que explica como decisores de topo devem ser estudados como unidade de análise, a fim de compreender o comportamento da empresa (Elron, 1997). O Top Management Team (TMT), assim, compreende os componentes de direção da organização. Nele está a base e o detalhamento da estratégia, metas, objetivos, missão e definição de responsabilidades que mantêm a organização se movendo na direção desejada (Alderson, 1993).

O TMT é definido como aquele composto por altos executivos que planejam e implementam a estratégia empresarial, de vital importância em todo o processo de gestão estratégica (Hao \& Yue, 2012). 
Os membros da equipe geralmente contêm o presidente, vice-presidente, executivo-chefe, gerentes gerais, os quais são responsáveis pelas atividades e pelos benefícios econômicos empresariais (Ping, Feng, \& Yanyan, 2016). Os gestores de topo concentram-se no plano de desenvolvimento a longo prazo, questões estratégicas e principais políticas. Eles podem controlar o pessoal e os recursos, mas têm como principal função a tomada de decisões (Yu-Hong, Bing, \& Yuan, 2013).

A equipe é o principal fórum de diálogo estratégico. É o meio no qual a discussão estratégica ocorre, as decisões são tomadas e implementadas e o futuro da organização é decidido. A qualidade e o nível de discussão estratégica e o futuro da organização, dependem da qualidade da interação entre os membros da equipe (Alderson, 1993). Devido à importante posição do TMT, a qualidade da decisão tomada pelos gestores pode impactar mais o desempenho da empresa do que as decisões tomadas por qualquer um dos gerentes comuns. A pesquisa sobre o processo de tomada de decisão do TMT é a favor de aprender acerca das características da equipe e da razão pela qual a sua heterogeneidade influencia o efeito da tomada de decisão. Podem ao mesmo tempo melhorar a eficiência da equipe e, consequentemente, da organização (Carpenter, Geletkanycz, \& Sanders, 2004).

Hambrick e Mason (1984) organizaram uma lista não esgotada de características gerenciais observáveis incluindo idade, experiências funcionais, experiências de carreira, educação, raízes socioeconômicas, posição financeira e características de grupo. Igualmente, indicaram 21 proposições associando essas características às escolhas estratégicas e ao desempenho dos resultados organizacionais. Essas proposições foram categorizadas em sete grupos: faixa etária relacionada à idade, outras experiências de carreira, educação formal, contexto socioeconômico, situação financeira e heterogeneidade de grupo (Chuang et al., 2007). Na sequência, pesquisas que levaram em conta os níveis médios da equipe sugerem que as principais características executivas como a idade (Goll, Johnson, \& Rasheed, 2008), nível educacional (Díaz-Fernández, González-Rodríguez, \& Pawlak, 2014; DíazFernández, González-Rodríguez, \& Simonetti, 2015), tempo no cargo (Pegels \& Yang, 2000; Gu, 2008), e a heterogeneidade (Li, Zhang, \& Zhang, 2015) estão associadas a decisões e resultados estratégicos.

\subsection{Estrutura de capital}

\subsubsection{Teorias da estrutura de capital}

A estrutura de capital de uma empresa concebe a maneira como está composto seu passivo. O tema é caracterizado por diversas teorias, cujas fundamentais são Modigliani e Miller, Trade-off $e$ Pecking Order. Modigliani e Miller (1958) são as referências iniciais da maior parte das pesquisas que visam esclarecer quais são os fatores determinantes da estrutura de capital das empresas. A argumentação inicial de que a forma de financiamento não implica, isto é, não é determinante do valor da empresa, e a posterior verificação do contrário, causou o surgimento de teorias que se utilizaram de elementos da realidade empresarial para explorar os fatores que elucidariam o financiamento das firmas (Tarantin Junior \& Valle, 2015). 
A Teoria de Modigliani e Miller anseia explicar os determinantes que influenciam a estrutura de capital das empresas, aproveitando-se da aplicação do conceito de equilíbrio de mercado de arbitragem, em que o valor de mercado de uma empresa é independente da sua estrutura de capital. De acordo com Modigliani e Miller (1958), empresas iguais possuem o mesmo valor independentemente de sua estrutura de financiamento ser fundamentada em capitais próprios ou de terceiros. Desta maneira, a estrutura de capital não é decisiva no valor da empresa e a decisão de investimento independente da origem dos recursos de terceiros.

A Teoria do Trade-off indica a existência de uma estrutura de capital ótima, todavia que não é suficiente para explicar a correlação entre alta lucratividade e baixos índices de endividamento. Nessa linha, não basta dizer (sem mais explicações) que os gestores são "excessivamente conservadores" ou "não maximizam o valor", culpá-los, em vez dos economistas, pelo fracasso da teoria dos economistas. Ademais, uma análise das táticas de financiamento rapidamente descarta a ideia de que os gestores não dão atenção aos impostos (Myers, 2001).

Com base na Teoria do Trade-off, organizações mais arriscadas, ou seja, aquelas com os maiores custos esperados de dificuldades financeiras, prevalecem-se menos do benefício fiscal advindo do acréscimo de dívidas em sua estrutura de capital, sendo, assim, menos alavancadas (Tarantin Junior \& Valle, 2015). A Teoria Pecking Order tem um de seus pilares no estudo de Ross feito em 1977, o qual avaliou a assimetria de informação entre gestores e investidores sobre a distribuição de dividendos, uma vez que esta política de distribuição sinaliza para o mercado as intenções futuras de investimento (Kayo, 2002). A teoria aprofundou-se com as pesquisas de Myers e Majluf (1984), que consideram a empresa como detentora de ativos já estabelecidos e oportunidades de crescimento e onde a estrutura de capital é função da mudança relativa destes dois elementos que formam a empresa.

A rentabilidade indica que empresas mais rentáveis, por ter maior quantia de recursos gerados por suas atividades, procuram menos financiamento externo, impedindo os problemas derivados da informação assimétrica, em linha com a Teoria de Pecking Order. Já que tais empresas não necessitam de amplas quantias de financiamento externo, elas teriam poucas oportunidades de dissolver os maiores custos fixos relacionados aos recursos de maiores maturidades, captando, consequentemente, de menores maturidades (Tarantin Junior \& Valle, 2015).

\subsubsection{Indicadores de estrutura de capital}

$\mathrm{Na}$ estrutura de capital o cálculo dos indicadores inclui, dentre outros, o Custo do Capital de Terceiros (CCT), o Custo do Capital Próprio (CCP) e o Custo Médio Ponderado do Capital (CMPC). O Custo do Capital de Terceiros (CCT) ou da dívida é o custo atual depois do imposto de renda do financiamento de longo prazo, por meio de empréstimos (Gitman, 2010). Em outras palavras, é o índice que avalia o custo atual para a empresa captar recursos para financiamento de projetos (Damodaran, 2012). 
Damodaran (2012) destaca que o CCT não é a taxa do cupom dos bônus que a firma tem a pagar, nem a taxa a qual a empresa obteve ao conseguir um financiamento no passado. Embora tais taxas possam ajudar na determinação do custo dos juros que a empresa terá de pagar, não definem o CCT depois do pagamento de impostos. Logo, uma empresa que contabilizou uma dívida contraída quando as taxas de juros estavam baixas, não pode informar que tem um CCT baixo se o nível geral das taxas de juros ou seu risco de inadimplência elevou no período.

Pelo aspecto temporal, os financiamentos podem ser de curto ou de longo prazo. Prazo maior provoca risco maior. No caso das dívidas de curto prazo, os valores contábeis e os valores de mercado tendem a ser similares, o que permite utilizar os próprios valores contábeis como equivalentes aos valores de mercado (Ross, 2007).

O Custo do Patrimônio Líquido (CCP) é o indicador de retorno que os investidores exigem para realizar um investimento patrimonial em uma companhia. Este retorno é igual ao que poderia ser obtido em outras aplicações de igual risco de mercado (Damodaran, 2012). O CCP é de cálculo mais difícil, pois não existe uma forma de constatar diretamente o retorno exigido pelos investidores em ações de uma firma (Ross, 2007). A estimativa do CCP é feita de acordo com modelos sugeridos na literatura de financeira. O modelo de avaliação com crescimento constante (modelo de Gordon) e o modelo de formação de preços de ativos (MFPA) são exemplos para cálculo do CCP (Gitman, 2010).

O Custo Médio Ponderado do Capital (CMPC) é a taxa de retorno exigida em qualquer proposta de investimento que possui o mesmo nível de risco que os ativos presentes na empresa. Precisa ser ajustado para mais ou para menos, a depender do tipo de projeto (por exemplo, projetos de substituição são de menor risco, já os de expansão ou novos são de maior risco) ou para diferentes unidades organizacionais (Bennouna, Meredith \& Marchant, 2010). Em outras palavras, o CMPC pode ser determinado como a média ponderada dos custos dos diversos componentes de financiamento, abrangendo dívida e patrimônio líquido, utilizados por uma organização para financiar suas necessidades financeiras (Damodaran, 2012). Calcula-se multiplicando o custo específico de cada modalidade de financiamento por seu percentual da estrutura de capital da empresa e, finalmente, somam-se os valores ponderados (Gitman, 2010).

De maneira geral, recomenda-se que as empresas apliquem taxas distintas para os projetos de investimentos, unidades ou divisões organizacionais. Ao sopesar o retorno de mercado, a empresa pode desenvolver taxas diferenciadas para os investimentos, até mesmo para projetos fora de seu negócio principal (Damodaran, 2012). 


\section{Procedimentos metodológicos}

\subsection{Hipóteses da pesquisa}

O nível médio de idade do Top Management Team (TMT) foi sugerido para ser relacionado a tendências como receptividade à mudança, pretensão de adotar novas ideias, busca de novidade, tomada de risco e flexibilidade. Essas inclinações, por seu turno, estão associadas à inovação estratégica, à extensão da diversificação das empresas, ao nível de expansão internacional, ao crescimento da empresa, entre outros. A consideração subjacente é que à medida que a idade média do TMT aumenta, a equipe se torna mais resistente à tomada de risco e mudança (Acar, 2016).

Gestores mais jovens podem ter menos compromisso com o status quo e, por conseguinte, estar mais propensos a utilizar estratégias novas e sem precedentes (Hambrick \& Mason, 1984). Por conta do compromisso com o status quo, um TMT composto por membros mais velhos pode ser mais restiva em tentar novas ideias ou admitir riscos (Koufopoulos, Zoumbos, Argyropouloz, \& Motwani, 2008). De outro viés, os gestores mais velhos já constituíram suas redes sociais, estilos de vida e planejamento de carreiras e tendem a se tornar aversivos ao risco (Chuang et al., 2009). Equipe mais antiga com a inclinação a ser avessa ao risco e com segurança financeira e profissional tende a escolher uma estrutura de capital mais conservadora (Chen et al., 2010). Assim, desenvolve-se a seguinte hipótese:

H1: A idade média dos gestores está positivamente relacionada com o nível de capital de terceiros das empresas.

As experiências perceptíveis dos gestores são proxies válidas para sua orientação cognitiva, valores e conhecimentos que em seguida impactarão suas escolhas estratégicas. Gestores com mais experiências internacionais, educação e experiência funcional diversificada tendem a tomar decisões para diminuir os riscos se comparados aos gestores com menos experiência e educação ou experiência funcional semelhante. Os gestores do alto escalão que detêm as referidas características tomam decisões estratégicas eficazes, como a avaliação completa de diversas opções para novas oportunidades, mitigando riscos e incertezas (Bany-Ariffin, Mcgowan, Júnior Tunde, \& Shahnaz, 2014).

A Teoria dos Escalões Superiores (TES) sugere uma relação linear positiva entre o nível de educação dos executivos e a base de conhecimento, a complexidade cognitiva, a abertura à mudança, a tolerância para a ambiguidade e a propensão ao risco. Nível educacional mais elevado é indicativo de maior nível de conhecimentos (Herremann \& Datta 2005). Um maior nível de conhecimento, por seu lado, deverá estar relacionado a estratégias mais eficazes e a uma gestão mais eficiente da organização.

Espera-se que equipes mais instruídas tenham conhecimento mais avançado e maior capacidade cognitiva. Isso, por sua vez, poderia expandir sua capacidade de tolerar ambiguidade, absorver novas informações, além de analisar, resolver e implementar soluções para problemas complexos. 
Nomeadamente, as TMTs com mais escolaridade seriam menos predispostas a escolher uma estrutura de capital conservadora (Chen et al., 2010).

Com isso, executivos com maior nível educacional podem ser mais confiantes em suas decisões de investimento e não carecerem de tanta folga financeira se comparados àqueles com nível educacional menor. Consequentemente, gestores com maior nível escolar serão menos tendentes a escolher uma estrutura de capital conservadora para o financiamento do investimento (Bortoluzzi, Zakaria, Santos, \& Lunkes, 2016). Nesse contexto, tem-se a segunda hipótese:

H2: O nível médio educacional está negativamente relacionado com o nível de capital de terceiros das empresas.

O tempo no cargo é uma característica que pode significar coesão social, a influência organizacional e o compromisso com o status quo, bem como a inércia e a falta de inovação (Bantel \& Jackson, 1989; Michel \& Hambrick, 1992). De tal modo, o tempo do cargo do TMT pode estar relacionado com o compromisso com o status quo e baixa criatividade (Wiersema \& Bantel, 1992; Hoffman, Williams, Lamont, \& Geiger, 2000). A longa duração média de tempo no cargo procede em níveis decrescentes de comunicação global, pois os membros do grupo sentem que são capazes de antecipar os pontos de vista de outros membros e acontece o acréscimo da especialização (Wiersema \& Bantel, 1992). Assim sendo, o longo mandato do TMT pode levar a um maior isolamento dos membros em relação a fontes externas de informação, o que pode reduzir a receptividade à mudança e à inovação (Camelo, Fernández-Alles \& Hernández, 2010).

Executivos com menos tempo de experiência na empresa podem estar mais propensos a assumir riscos, pois são obrigados a produzir resultados e a se mostrarem como gestores competentes. Gestores com mais tempo, por sua vez, podem ter uma abordagem mais avessa ao risco, uma vez que são menos pressionados a se comprovarem (Kor, 2006). Isto posto, desenvolve-se a terceira hipótese:

H3: O tempo médio no cargo está negativamente relacionado com o nível de capital de terceiros das empresas.

\subsection{Amostra e coleta de dados}

A população do estudo é constituída de 114 empresas do setor Financeiro listadas na Brasil, Bolsa, Balcão $\left(\mathrm{B}^{3}\right)$, bolsa de valores oficial do Brasil, em 31 de julho de 2019. Do total, 19 empresas estrangeiras não disponibilizaram as informações, perfazendo uma amostra de 95 empresas. Os dados foram coletados no endereço eletrônico da $\mathrm{B}^{3}$ entre os dias 11 de julho a 31 de julho de 2019 referentes ao exercício de 2018. 
Quadro 1 - Composição da amostra

\begin{tabular}{|l|c|c|}
\hline \multicolumn{1}{|c|}{ Subsetor } & Qetor Financeiro & Estrangeiras \\
\hline Exploração de Imóveis & 17 & $(1)$ \\
\hline Holdings Diversificadas & 5 & (7) \\
\hline Intermediários Financeiros & 32 & $(3)$ \\
\hline Previdência e Seguros & 8 & $(4)$ \\
\hline Securitizadoras de Recebíveis & 19 & $(1)$ \\
\hline Serviços Financeiros Diversos & 8 & $(3)$ \\
\hline Outros & 25 & $(19)$ \\
\hline Total & 114 & \\
\hline
\end{tabular}

Fonte: Brasil, Bolsa, Balcão (2018).

A coleta dos dados foi efetuada em dois momentos. A primeira envolveu a coleta dos das variáveis independentes de idade, nível educacional e tempo no cargo dos diretores das empresas, por meio dos formulários de referência de 2018, no tópico de assembleia e administração. O Quadro 2 apresenta as variáveis independentes:

Quadro 2 -Variáveis independentes

\begin{tabular}{|c|c|l|}
\hline Variável independente & Abreviação & \multicolumn{1}{c|}{ Forma de cálculo } \\
\hline Idade média & IM & Média da idade dos gestores da equipe \\
\hline Nível médio educacional & NME & $\begin{array}{l}\text { Nível médio ou menos }=0 \text {, Graduação = 1, Pós- } \\
\text { graduação ou Master Business Administration } \\
(\text { MBA })=2, \text { Mestrado }=3, \text { Doutorado }=4 .\end{array}$ \\
\hline Tempo médio no cargo & TMC & Média de meses no cargo dos membros da equipe \\
\hline
\end{tabular}

Fonte: Autores.

A segunda etapa corresponde à coleta dos dados financeiros presentes no Balanço Patrimonial de 2018 das companhias, Exigível total ou Passivo Não Circulante e Patrimônio líquido, os quais foram obtidos por meio das Demonstrações Financeiras Padronizadas (DFP) divulgadas pelas empresas também no sítio eletrônico da $\mathrm{B}^{3}$. A variável dependente Nível de Capital de Terceiros (NCT) é calculada pela divisão do Exigível Total pelo Patrimônio Líquido ((Passivo Circulante + Passivo Não Circulante)/Patrimônio Líquido). A seguir apresenta-se a equação do modelo:

$$
N C T=\partial+\beta_{1} \mathfrak{I}+\beta_{2} N M E+\beta_{3} T M C+\mu
$$

em que $\partial$ é a constante da função, $\beta_{1}, \bigcap_{2} e ß_{3}$ são os coeficientes estimados da regressão e $\mu$ é o erro da regressão. 
Os dados são tratados com o uso do procedimento estatístico regressão linear e regressão linear múltipla em Mínimos Quadrados Ordinários (MQO). Para tanto empregou-se o software Gretl. Inicialmente foi realizada a correlação linear entre as variáveis e, na sequência, a regressão múltipla.

\section{Resultados e análises dos dados}

As informações analisados são referentes a 699 pessoas de 95 empresas ou Top Management Team (TMT). A média de diretores por equipe é aproximadamente de 7,36, sendo mediana 5, mínimo 2 e máximo 44 integrantes. A Tabela 1 apresenta as características dos gestores que compõem os TMTs.

Tabela 1 - Estatística descritiva da amostra (95 empresas)

\begin{tabular}{c|c|c}
\hline Variáveis & \multicolumn{2}{|c}{ Frequência } \\
\hline Idade & Média & Desvio Padrão \\
\hline Tempo no cargo & 49,83 & 11,06 \\
\hline Nível educacional & 61,67 & 42,48 \\
\hline Nível Médio & 1,38 & 0,62 \\
\hline Graduação & Frequência Relativa & Frequência \\
\hline Pós-graduação ou MBA & 94 & $13,43 \%$ \\
\hline Mestrado & 272 & $38,85 \%$ \\
\hline Doutorado & 244 & $34,94 \%$ \\
\hline
\end{tabular}

Fonte: Dados da pesquisa.

A análise descritiva possibilita observar que as 95 organizações da amostra possuem gestores com média de idade de 50 anos e 5 anos de tempo no cargo. No que cerne ao nível educacional, a maioria possui somente graduação ou também pós-graduação/MBA. A realização de Mestrado ou Doutorado ainda alcança menos de $15 \%$ dos diretores das companhias financeiras.

Quanto à estrutura de capital, percebeu-se que em média $73 \%$ são provenientes de terceiros, enquanto que $27 \%$ são oriundos de capital próprio. Em outras palavras, as sociedades da amostra tendem a recorrer para recursos de terceiros, de maneira geral, para sustentação de suas atividades. Previamente ao exame das hipóteses, decorre-se a verificação de valores outliers na amostra. Nesse passo, verificase a presença de valores outliers de endividamento. Identificaram-se 3 empresas com tais valores discrepantes acima ou abaixo dos limites superior e inferior da amostra. Para que referidos dados não distorçam a análise global dos resultados, foram eliminados, perfazendo um total de 92 observações (empresas do setor Financeiro). 
Efetivam-se testes de multicolinearidade dos dados e correlação de Pearson para confirmar a normalidade dos dados. O Quadro 2 expõe a matriz de correlação linear, o qual concebe o grau de associação entre as variáveis. Consideram-se as variáveis correlacionadas quando a alteração em uma implica em variações na(s) outra(s) variável(eis). O coeficiente pode alternar de -1(correlação negativa) $\mathrm{a}+1$ (correlação positiva). Valor zero significa ausência de correlação, mais próximo a +1 significa correlação positiva forte e a -1 correlação negativa forte.

Tabela 2 - Matriz de correlação (92 empresas)

\begin{tabular}{c|c|c|c|c}
\hline Variáveis & IM & NME & TMC & NCT \\
\hline IM & 1 & 0,0069 & 0,3616 & 0,2435 \\
\hline NME & - & 1 & $-0,0178$ & $-0,2120$ \\
\hline TMC & - & - & 1 & $-0,0731$ \\
\hline NCT & - & - & - & 1 \\
\hline
\end{tabular}

Fonte: Dados da pesquisa.

Os valores obtidos mostram que as associações de Nível Médio Educacional com Tempo Médio no Cargo e com Nível de Capital de Terceiros são negativas (opostas). Isto quer dizer que, quanto maior o Nível Educacional dos membros da equipe, menor tende a ser o Tempo Médio no Cargo e o Nível de Capital de Terceiros da empresa. A maior correlação é entre a Idade Média e o Tempo médio no cargo (36,16\%); mas mesmo assim ainda é fraca.

O teste das hipóteses desenvolvidas nessa pesquisa é feito por meio de modelos de regressão linear múltipla e simples. A Tabela 3 inclui os resultados de três modelos, os quais foram testados com o desígnio de demonstrar o que melhor explica as variáveis. 
Tabela 3 - Modelos de regressão linear

\begin{tabular}{l|c|c|c|c|c}
\hline & Variável & Coeficiente & Erro padrão & Razão-t & P-valor \\
\hline Modelo 1 & const & 0,214290 & 5,85812 & 0,03658 & 0,9710 \\
\hline R-quadrado = 0,136482 & IM & 0,229716 & 0,111244 & 2,065 & $0,0450 * *$ \\
\hline $\begin{array}{l}\text { R-quadrado ajustado }= \\
0,076237\end{array}$ & NME & $-2,84029$ & 1,85057 & $-1,535$ & 0,1322 \\
\hline P-valor(F) $=0,094449$ & TMC & $-0,0363332$ & 0,0290036 & $-1,253$ & 0,2171 \\
\hline
\end{tabular}

\begin{tabular}{l|c|c|c|c|c}
\hline Modelo 2 & Variável & Coeficiente & Erro padrão & Razão-t & P-valor \\
\hline R-quadrado = 0,104968 & const & 0,416707 & 5,89366 & 0,07070 & 0,9440 \\
\hline $\begin{array}{l}\text { R-quadrado ajustado }= \\
0,064285\end{array}$ & IM & 0,179295 & 0,104376 & 1,718 & $0,0929 *$ \\
\hline P-valor(F) $=0,087186$ & NME & $-2,78980$ & 1,86206 & $-1,498$ & 0,1412 \\
\hline
\end{tabular}

\begin{tabular}{l|c|c|c|c|c}
\hline Modelo 3 & Variável & Coeficiente & Erro padrão & Razão-t & P-valor \\
\hline R-quadrado $=0,059307$ & const & $-3,36829$ & 5,39789 & $-0,6240$ & 0,5358 \\
\hline $\begin{array}{l}\text { R-quadrado ajustado }= \\
0,038403\end{array}$ & IM & 0,178218 & 0,105807 & 1,684 & $0,0990 *$ \\
\hline P-valor(F) $=0,099036$ & & & & & \\
\hline
\end{tabular}

Fonte: Dados da pesquisa.

Os três modelos foram estimados por meio de Mínimos Quadrados Ordinários. Ao verificar os valores dos coeficientes estimados para as variáveis independentes do Modelo 1, verifica-se que IM (Idade Média dos gestores da equipe) foi a variável que exibiu significância estatística a um nível de 5\% $(p$-valor $=0.0450)$. O resultado do R-quadrado significa que o referido modelo explica perto de $13,65 \%$ do nível de capital de terceiros da empresa.

Na estimação do Modelo 2 foi suprimida a variável Tempo médio no cargo dos diretores do alto escalão, uma vez que apresentou o p-valor mais alto (0.2171). Assim, houve redução no poder explicativo do Modelo, se comparado ao Modelo 1, tendo em vista que um variável explicativa foi eliminada. Contudo, a significância do Modelo 2 é mais alta ( $p$-valor $=0,087186)$.

Na mesma linha, extinguiu-se no Modelo 3 a variável Nível médio educacional dos gestores da equipe por não oferecer significância estatística no Modelo 2. Ocorre que com isso o R2 ajustado apresentou redução de 10,5\% para 5,93\%. O Modelo 3 apresenta significância estatística menor se compara ao Modelo 2, com p-valor = 0,099036. Destarte, o segundo modelo é o que melhor exemplifica a variação do endividamento, mais precisamente, o Nível de capital de terceiros é NCT $=0,416707+$ $0,179295 * \mathrm{IM}-2,78980 * \mathrm{NME}$.

Como conclusão, é possível constatar que a idade média dos gestores do TMT afeta positivamente a participação de capital de terceiros das empresas do setor financeiro da amostra. Neste 
sentido, é possível aceitar a primeira hipótese de que a idade média dos gestores está positivamente relacionada com o nível de capital de terceiros das empresas. Em consonância com a literatura, equipe mais antiga tende a ser avessa ao risco e e a escolher uma estrutura de capital mais conservadora (Chen et al., 2010).

A hipótese 2 a qual descreve que o nível médio educacional está negativamente relacionado com o nível de capital de terceiros das empresas., não foi estatisticamente comprovada. Não obstante, tal achado permite a interpretação, com base nos Modelos 1 e 2, de que o nível médio educacional da equipe é negativamente associado com a variável nível de capital de terceiros (endividamento) das empresas financeiras. Os resultados encontrados vão ao encontro das ponderações de Bantel e Jackson (1989), Wiersema e Bantel (1992), e Barker e Mueller (2002) e contrário a Bortoluzzi et al. (2016).

A terceira hipótese de que o tempo médio no cargo está negativamente relacionado com o nível de capital de terceiros das empresas não foi significativamente sustentada. Acredita-se que a elevada variação da média de idade entre as equipes não tenha favorecido a correlação com a variação do nível de capital de terceiros das respectivas empresas. Contudo, nota-se pelo Modelo 1 que o tempo médio no cargo do TMT está negativamente relacionada ao nível de capital de terceiros. Isso porque gestores com menos tempo de experiência na empresa podem estar mais propensos a assumir riscos, pois são obrigados a produzir resultados e a se mostrarem como gestores competentes.

Gestores com mais tempo, por sua vez, podem ter uma abordagem mais avessa ao risco, uma vez que são menos pressionados a se comprovarem (Kor, 2006). Por estarem mais comprometidos com o status quo da empresa, o tempo no cargo é um indicativo de que gestores mais experientes no cargo de gestão tendem a diminuir o uso de recursos de terceiros em prol do capital próprio.

\section{Conclusões}

O trabalho de Hambrick e Mason (1984) demonstrou que as características do Top Management Team (TMT) eram fatores preditivos para explicar o desempenho das organizações. Na sequência, pesquisas confirmaram que características observáveis, como idade, sexo, educação e experiência funcional são indicativos de aspectos gerenciais cognitivos e afetivos subjacentes que determinam as decisões das equipes de gestão, que afetam posteriormente o desempenho da organização (Bell et al., 2011).

Diferentes pesquisas concentraram-se em Top Management Team (TMT) e seu impacto em decisões estratégicas. O efeito do TMT sobre o desempenho da empresa, em especial, tem sido de interesse contínuo para os estudiosos da área estratégica (Koufopoulos et al., 2008). Diversas variáveis como idade (Chuang et al., 2009, 2010), tempo no cargo (Gu, 2008) ou nível educacional (Camelo, Fernández-Alles \& Hernández, 2010) têm sido utilizadas para analisar a influência das equipes.

Nesse contexto, a finalidade deste estudo foi analisar a relação entre as características do Top Management Team e a estrutura de capital das empresas financeiras listadas na Brasil, Bolsa, Balcão em 
2018. Por meio da análise estatística pode-se constatar que idade média dos gestores do TMT tem uma relação positiva significativa com o nível de capitais de terceiros das companhias. Igualmente, uma maior maturidade dos membros da equipe pode torná-lo mais arrojado em relação ao endividamento da empresa.

No que diz respeito às características nível educacional médio e tempo médio no cargo do TMT, observou-se ausência de significância estatística. Ainda assim, ambas se mostraram negativas em relação à ao endividamento das empresas. Ou seja, quanto maior a média do nível educacional e do tempo no cargo do TMT espera-se um menor nível de capital de terceiros na estrutura de capital da empresa.

A realização da presente pesquisa, além de contribuir para a evolução do tema proposto, aumenta a compreensão da relação entre as características da gestão de topo e a maneira como é composta a estrutura de capital na prática das empresas. É possível utilizar os resultados para aplicação nos processos de recrutamento e seleção. Dessa maneira, ao estabelecer as características dos profissionais para atuarem na gestão de capital, as empresas tenham em mente a estrutura de capital que melhor se relaciona com o perfil do TMT. A idade média do TMT inclina-se a ter relação significativa positiva com o nível de capital de terceiros da empresa, enquanto o tempo médio no cargo e nível médio educacional do TMT tende a ter relação negativa com o endividamento das empresas.

O presente estudo apresenta algumas limitações, como por exemplo, a amostra, o período de análise e a quantidade de características observáveis do TMT. Igualmente, os achados alcançados não podem ser generalizados a outros grupos de empresas de outros setores e períodos. Sugere-se que pesquisas futuros analisem diferentes períodos de tempo, além do impacto de outras características observáveis do TMT, como por exemplo, experiência internacional, gênero ou dualidade dos membros nas equipes. Adicionalmente, avaliem a relação dessas características com os diferentes níveis de capital próprio e de terceiros, de investimento em pesquisa e desenvolvimento e de inovação.

\section{Referências}

Acar, F. P. (2016) The effects of top management team composition on SME export performance: an upper echelons perspective. Central European Journal of Operations Research, 24, 833-852.

Ahmad, R., ArifF, M., \& Skully, M. (2008) The determinants of bank capital ratios in a developing economy. Asia-Pacific Financial Markets, 15(3), 255-272.

Alderson, S. (1993) Reframing management competence: focusing on the Top Management Team. Personnel Review, 22(6), 53-62.

Bantel, K. A., \& Jackson, S. E. (1989) Top management and innovations in banking: does the composition of the top management team make a difference? Strategic Management Journal, 10, 107-124. 
Bany-Ariffin, A. N., McGowan, C. B., Júnior Tunde, M. B., \& Shahnaz, A. S. (2014) Top management characteristics and firm's international diversification activities: evidence from a Developing Nation. Pertanika Journal of Social Sciences \& Humanities, 22, 39-54.

Barker, V., \& Mueller, G. (2002) CEO characteristics and firm R\&D spending. Management Science, v. 48(6), 782-801.

Bennouna, K., Meredith, G. G., \& Marchant, T. (2010) Improved capital budgeting decision making: evidence from Canada. Management Decision, v. 48(2), 225-247.

Bell, S. T.; Villado, A. J.; Lukasik, M. A.; Belau, L.; Briggs, A. L. (2011) Getting specific about demographic diversity variable and team performance relationships: a meta-analysis. Journal of Management, 37(3), 709-743.

Bertrand, M., \& Schoar, A. (2003) Managing with style: The effect of managers on firm policies. The Quarterly Journal of Economics, 118(4), 1169-1208.

Bortoluzzi, D. A., Zakaria, J., Santos, E. A., \& Lunkes, R. J. (2016) A influência das características dos executivos de alto escalão sobre a estrutura de capital: Um estudo em empresas listadas na BM\&FBOVESPA. Espacios, 37(37), 28.

Cabrera-Suárez, M. K., \& Martín-Santana, J. D. (2013) Top management teams and performance in non-listed family firms. Journal of Management \& Organization, v. 19, p. 405-423, 2013.

Camelo, C., Fernández-Alles, M., \& Hernández, A. B. (2010) Strategic consensus, top management teams, and innovation performance. International Journal of Manpower,31(6), 678-695.

Carpenter, M. A., Geletkanycz, M. A., \& Sanders, G. W. (2004) Upper echelons research revisited: antecedents, elements, and consequences of top management team composition. Journal of Management, 30(6), 749-778.

Chen, H-.L., Hsu, W.-T., \& Huang, Y.-S. (2010) Top management team characteristics, R\&D investment and capital structure in the IT industry. Small Business Economics, 35, 319-333.

Chenhall, R. H. (2003) Management control systems design within its organizational context: Findings from contingency-based research and directions for the future. Accounting, Organizations and Society, 28(2-3), 127-168.

Chevalier, J. (1995) Capital structure and product market competition: Empirical evidence from the supermarket industry. American Economic Review, 85, 415-435.

Chuang, T. T., Nakatani, K., \& Zhou, D. An exploratory study of the extent of information technology adoption in SMEs: an application of upper echelon theory. Journal of Enterprise Information Management, v. 22, n. 1/2, p. 183-196, 2009.

Chuang, T. T., Nakatani, K., \& Zhou, D. (2007) The impact of managerial characteristics of top management team on the extent of information technology adoption: An exploratory study with the upper echelon theory. Anais do Americas Conference on Information Systems, Praga, 13.

Cyert, R. M., \& March, J. G. (1963) A behavioral theory of the firm. New Jersey: Prentice-Hall.

Damodaran, A. (2002) Investment evaluation: tools and techniques for determining the value of any asset. 3. ed. New York: John Wiley \& Sons, 2002. 
Díaz-Fernández, M. C., González-Rodríguez, M. R., \& Pawlak, M. (2014) Top management demographic characteristics and company performance. Industrial Management and Data Systems, 114(3), 365- 386.

Díaz-Fernández, M. C., González-Rodríguez, M. R., \& Simonetti, B. (2015) Top Management Teams' demographic characteristics and their influence on strategic change, Quality \& Quantity, 49, 13051322.

Elron, E. (1997) Top Management Teams within Multinational Companies: Effects of Cultural Heterogeneity . Journal of International Business Studies, 8(4), 393-412.

Gitman, L. J. (2010) Principles of managerial finance (12a ed.). New Jersey: Prentice Hall.

Goll, I., Johnson, N. B., \& Rasheed, A. (2008) Top management team demographic characteristics, business strategy, and firm performance in the US airline industry - The role of managerial discretion. Management Decision, 46(2), 201-222.

Gropp, R., \& Heider, F. (2010) The determinants of bank capital structure. Review of Finance, 14(4), 587-622.

Gu, J. (2008) An empirical study on the relation between characteristics of enterprise Top Management Team and strategic decision in industry cluster of China. International Journal of Human Resources Development and Management, 8(1/2), 96-110.

Hambrick, D. C. (2007) Upper echelons theory: an update. The Academy of Management Review, 32(2), 334-343.

Hambrick, D. C; \& Mason, P. A. (1984) Upper echelons: The organization as a reflection of its top managers. Academy of Management Review, 9, 193-206.

Hanousek, J., \& Shamshur, A.( 2011) A stubborn persistent: is the stability of leverage ratios determined by the stability of the economy. Journal of Corporate Finance, 17(5), 1360-1376.

Hao, Y., \& Yue, C. T. (2012) The influence of strategic orientation on innovation performance: A research framework from TMT perspective. In: INTERNATIONAL SYMPOSIUM ON MANAGEMENT OF TECHNOLOGY, Hangzhou. Anais... Hangzhou.

Herrmann, P., \& Datta, D. K. (2005) CEO experiences: Effects on the choice of FDI entry mode. Journal of Management Studies, 43(4), 755-778.

Hiebl, M. R. W. (2014) Upper echelons theory in management accounting and control research. Journal of Management Control, 24(3), 223-240.

Hoffman, J. J.; Williams, R. J.; Lamont, B. T.; \& Geiger, S. W. (2000) Managerial tenure and recovery following M-form reorganization in diversified firms. Journal of Business Research, 50, 287-296.

Kayo, E. K. (2002) A estrutura de capital e o risco das empresas tangível e intangível-intensiva: uma contribuição ao estudo da valoração de empresas. 110 f. Tese (Doutorado em Administração) Faculdade de Economia, Administração e Contabilidade. Universidade de São Paulo. São Paulo.

Koufopoulos, D., Zoumbos, V., Argyropoulou, M., \& Motwani, J. (2008) Top management team and corporate performance: a study of Greek firms. Team Performance Management, v. 14(7/8), 340363. 
Kor, Y. Y. (2006) Direct and interaction effects of top management team and board compositions on R\&D investment strategy. Strategic Management Journal, 27(11), 1081-1099.

Lemmon, M. L., Roberts, M. R., \& Zender, J. F. (2008) Back to the beginning: Persistence and the cross-section of corporate capital structure. Journal of Finance, 63(4), 1575-1608.

Li, W., Zhang, S., \& Zhang, J. (2015) Top management team heterogeneity, diversification, and corporate performance: A panel smooth transition regression model. Romanian Journal of Economic Forecasting, 18(1), 129-142.

Matemilola, B. T., Bany-Ariffin, A. N., Azman-Saini, W. N. W., \& Nassir, A. M. (2018) Does top managers' experience affect firms' capital structure? Research in International Business and Finance, 45, 488-498.

Maksimovic, V. (1998) Capital Structure in Repeated Oligopolies. Rand Journal of Economics, 19(3), 389-407.

Michel, J. G., \& Hambrick, D. C. (1992) Diversification posture and top management team characteristics. Academy of Management Journal, 35(1), 9-37.

Modigliani, F., \& Miller, M. H. (1958) The cost of capital, corporate finance and the theory of investment. American Economic Review, 48(3), 261-297.

Mwangi, K. L. W., Makau, M. S., \& Kosimbei, G. (2014) Relationship between Capital Structure and Performance of NonFinancial Companies Listed In the Nairobi Securities Exchange. Global Journal of Contemporary Research in Accounting, Auditing and Business Ethics (GJCRA) An Online International Research Journal, 1(2), 72-90.

Myers, S. C. (2001) Capital structure. Journal of Economic Perspectives, 15(2), 81-102.

Myers, S. C., \& Majluf, N. S. (1984) Corporate financing and investment decisions when firms have information that investors do not have. Journal of Financial Economics, 13(2), p. 187-221.

Octavia, M., \& Brown, R. (2010) Determinants of bank capital structure in developing countries: regulatory capital requirement versus the standard determinants of capital structure. Journal of Emerging Markets, 15(1), 50-62.

Pegels, C. C. Yang, B. (2000) Top management team impact on strategic assets accumulation capabilities. Management Decision, 38(10), 694-710.

Ping, G.; Feng, W.; Yanyan, Z. (2016) A Review of Enterprise Top Management Team and Organizational Innovation. In: International Conference on Innovation and Management, 13., Wuhan. Anais... Wuhan.

Ross, S.A. (2007) Fundamentals of Corporate Finance, 4th ed., Sydney: McGraw Hill.

Strauß, E., \& Zecher, C. (2013) Management control systems: A review. Journal of Management Control, 23(4), 233-268.

Tarantin Junior, W., \& Valle, M. R. (2015) Capital structure: the role of the funding sources on which Brazilian listed companies are based. Revista Contabilidade \& Finanças, 26(69), 331-344.

Wang, Y. K. (2015) The impact of prestigious top management team on international alliance formation: Evidence from Taiwanese electronics firms. Journal of Management \& Organization, $21(6), 835-852$. 
Wiersema, M. P., \& Bantel, K. A. (1992) Top management team demography and corporate strategic change. Academy of Management Journal, 35, 91-121.

Xie, X. Y., Wang, W. L., \& Qi, Z. J. (2015) The effects of TMT faultine configuration on a firm's short-term performance and innovation activities. Journal of Management \& Organization, 21(5), $558-572$.

Yu-Hong, A.; Bing, L.; Yuan, L. (2013) Mechanism of action of TMT managerial discretion to strategic decision. In: INTERNATIONAL CONFERENCE ON MANAGEMENT SCIENCE AND ENGINEERING, 20., Harbin. Anais... Harbin. 